\title{
A Phase III study of zanubrutinib plus rituximab versus bendamustine plus rituximab in transplant-ineligible, untreated mantle cell lymphoma
}

\author{
Martin Dreyling ${ }^{*, 1}$, Constantine S Tam², Michael Wang ${ }^{3}$, Stephen D Smith ${ }^{4}$, Marco \\ Ladetto ${ }^{5}$, Huiqiang Huang ${ }^{6}$, William Novotny ${ }^{7}$, Melannie $\mathrm{Co}^{7}$, Alfredo Romano ${ }^{8}$, Eric \\ Holmgren ${ }^{7}$, Jane Huang ${ }^{7}$ \& Steven Le Gouill ${ }^{9}$ \\ ${ }^{1}$ LMU University Hospital, Munich, 81377, Germany \\ ${ }^{2}$ Peter MacCallum Cancer Centre, St Vincent's Hospital, Royal Melbourne Hospital, University of Melbourne, Melbourne, \\ Victoria 3000, Australia \\ ${ }^{3}$ The University of Texas MD Anderson Cancer Center, Houston, TX 77030, USA \\ ${ }^{4}$ University of Washington/Fred Hutchinson Cancer Research Center, Seattle, WA 98109, USA \\ ${ }^{5}$ Divisione di Ematologia, Azienda Ospedaliera SS. Antonio e Biagio e Cesare Arrigo, Alessandria, 15121, Italy \\ ${ }^{6}$ Sun Yat-sen University Cancer Center, Guangzhou, Guangdong Province, 510060, China \\ ${ }^{7}$ BeiGene USA, Inc., San Mateo, CA 94403, USA \\ ${ }^{8}$ BeiGene Switzerland $\mathrm{GmbH}$, 4051 Basel, Switzerland \\ ${ }^{9}$ Centre Hospitalier Universitaire de Nantes, 44093 Nantes, France \\ *Author for correspondence: Tel.: +89 440072 202; Martin.Dreyling@med.uni-muenchen.de
}

Mantle cell lymphoma is an aggressive B-cell malignancy. Current frontline chemoimmunotherapies produce high response rates but relapse is inevitable. Furthermore, the elderly and those with comorbidities are precluded from standard regimens and stem cell transplant, leaving them with limited options. Targeted therapies, including Bruton tyrosine kinase inhibitors, are an effective treatment strategy in mantle cell lymphoma. Zanubrutinib is a potent next-generation Bruton tyrosine kinase inhibitor that has demonstrated complete and sustained Bruton tyrosine kinase occupancy, minimal off-target effects and favorable pharmacokinetic/pharmacodynamic properties. Described herein is an ongoing Phase III study comparing the efficacy and safety of zanubrutinib plus rituximab followed by zanubrutinib monotherapy versus bendamustine plus rituximab followed by observation in transplant-ineligible patients with previously untreated mantle cell lymphoma.

Clinical Trial Registration: NCT04002297 (ClinicalTrials.gov)

First draft submitted: 4 August 2020; Accepted for publication: 4 September 2020; Published online: 28 September 2020

Keywords: Bruton tyrosine kinase $\bullet$ BTK inhibitor $\bullet$ clinical trial $\bullet$ mantle cell lymphoma $\bullet$ zanubrutinib

Here we describe an ongoing, head-to-head, randomized Phase III study comparing the efficacy of zanubrutinib (BGB-3111) and rituximab followed by zanubrutinib monotherapy with that of bendamustine plus rituximab followed by observation in patients with previously untreated mantle cell lymphoma (MCL) who are ineligible for stem cell transplantation (SCT) as measured by progression-free survival (PFS) determined by an independent review committee (IRC). Secondary end points include PFS determined by investigator assessment (IA), overall response rate (ORR), duration of response (DOR) and overall survival (OS). This study also includes an exploratory analysis of clinical outcomes according to prognostic and predictive biomarkers, pharmacokinetic (PK) parameters and mechanisms of resistance (ClinicalTrials.gov: NCT04002297).

\section{Background \& rationale}

MCL is one of the most challenging hematologic malignancies to manage owing to its aggressive clinical course and relative lack of effective cure. MCL accounts for $6 \%$ of all non-Hodgkin lymphoma (NHL) cases [1,2]. MCL is diagnosed in $0.51-0.55 / 100,000$ persons per year in the USA with a similar incidence rate worldwide [3,4].

Future $\because$ Medicine 


\begin{tabular}{|c|c|c|c|}
\hline Best response & Treatment naive $(n=11)$ & Relapsed / refractory $(n=37)$ & Efficacy evaluable $(n=48)$ \\
\hline $\begin{array}{l}\text { ORR, n (\%) } \\
(95 \% \mathrm{Cl})\end{array}$ & $\begin{array}{l}9(81.8) \\
(48.2-97.7)\end{array}$ & $\begin{array}{l}32(86.5) \\
(71.2-95.5)\end{array}$ & $\begin{array}{l}41(85.4) \\
(72.2-93.9)\end{array}$ \\
\hline $\begin{array}{l}\mathrm{CR}^{\dagger}, \mathrm{n}(\%) \\
(95 \% \mathrm{Cl})\end{array}$ & $\begin{array}{l}3(27.3) \\
(6.0-61.0)\end{array}$ & $\begin{array}{l}11(29.7) \\
(15.9-47.0)\end{array}$ & $\begin{array}{l}14(29.2) \\
(17.0-44.1)\end{array}$ \\
\hline PR, n (\%) & $6(54.5)$ & $21(56.8)$ & $27(56.3)$ \\
\hline DOR median, months $(95 \% \mathrm{Cl})$ & NE (9.2-NE) & $15.4(11.5-28.2)$ & $16.2(11.5-28.2)$ \\
\hline Follow-up median, months (range) & $8.3(1.6-27.9)$ & $19.4(1.9-38.2)$ & $16.7(1.6-38.2)$ \\
\hline
\end{tabular}

The median age at diagnosis is 70 years and it is three-times more common in men than in women $[5,6]$. The majority of patients present with advanced-stage disease, including lymphadenopathy, organomegaly and bone marrow involvement and they frequently require immediate treatment [2]. The pathognomonic feature of MCL is overexpression of cyclin D1, which is a consequence of juxtaposition of the proto-oncogene CCND1 on chromosome $11 \mathrm{q} 13$ to the immunoglobulin heavy chain gene at chromosome 14q32 [7].

Current frontline treatment usually includes chemo-immunotherapeutic options, most commonly rituximabcyclophosphamide-doxorubicin hyrdrochloride-vincristine-prednisolone or bendamustine-rituximab. While response rates are often high, with duration of response varying from 18-30 months [8], multiple relapses are common $[9,10]$. Younger, fit patients have the option of high-intensity induction chemotherapy, such as hyperfractionated cyclophosphamide, vincristine, doxorubicin, dexamethasone (hyper-CVAD) followed by SCT, which results in prolonged remission for many patients [11,12]. However, the elderly and those with comorbidities are precluded from standard chemo-immunotherapeutic regimens and SCT. These patients have limited options and treatment is largely palliative. No standard treatment approach has been defined for these patients [5].

Bruton tyrosine kinase (BTK) mediates B-cell proliferation, migration, adhesion and survival and is constitutively active in MCL [7,13]. A number of BTK inhibitors, including zanubrutinib, have shown activity in relapsed/refractory (R/R) MCL and are approved by the US FDA [14-16].

Zanubrutinib is a next-generation BTK inhibitor designed to maximize BTK occupancy. In preclinical studies, zanubrutinib has been shown to be a highly potent, selective, bioavailable and irreversible BTK inhibitor [17]. Zanubrutinib has greater selectivity for BTK versus off-target TEC- and EGFR-family kinases which are thought to cause adverse events (AEs) such as diarrhea, thrombocytopenia, bleeding, atrial fibrillation, rash and fatigue, which can be associated with BTK inhibitor therapy $[18,19]$. Complete and sustained BTK occupancy in both peripheral blood mononuclear cells and lymph nodes was achieved in all patients in the first-in-human Phase I study BGB-3111-AU-003 at the $160 \mathrm{mg}$ twice-daily therapeutic dose (ClinicalTrials.gov: NCT02343120) [17]. Achieving complete BTK inhibition in both blood and lymph nodes is hypothesized to help provide meaningful and sustained responses in MCL and other hematologic malignancies. Based on drug-drug interaction studies and $\mathrm{PK} /$ pharmacodynamic analyses, proton pump inhibitors or other acid-reducing agents do not affect zanubrutinib exposure [16]. Zanubrutinib may be coadministered with strong or moderate CYP3A inhibitors at a reduced dose. In addition, antiplatelet medications and anticoagulants including warfarin have been allowed on zanubrutinib trials. There is now extensive clinical experience at the $160-\mathrm{mg}$ twice-daily and 320-mg once-daily doses of zanubrutinib. Both dosing schedules have shown a high level of activity without compromising the tolerability profile.

Zanubrutinib monotherapy has been shown to be highly active and well tolerated in patients with MCL in two single-arm studies (BGB-3111-AU-003 [NCT02343120] and BGB-3111-206 [NCT03206970]) [20,21]. In BGB3111-AU-003, 53 patients with MCL (37 R/R and 16 treatment-naive) were dosed. The investigator-assessed ORR was $85.4 \%$ with a median DOR of 16.2 months (Table 1) [20]. The most common AEs were contusion (39.6\%), diarrhea (34\%), upper respiratory tract infection (26.4\%) and constipation and fatigue (22.6\% each). The majority of AEs were grade $1 / 2$, with anemia as the most common grade $\geq 3 \mathrm{AE}(9.4 \%)$. Ten patients (18.9\%) discontinued treatment due to AEs; major hemorrhage and atrial fibrillation/flutter occurred in four patients (7.5\%) [20]. 


\begin{tabular}{|c|c|}
\hline Best response & $\mathrm{n}=86$ \\
\hline $\begin{array}{l}\text { ORR, n (\%) } \\
(95 \% \mathrm{Cl})\end{array}$ & $\begin{array}{l}72(84) \\
(74-91)\end{array}$ \\
\hline CR, n (\%) & $59(68.6)$ \\
\hline PR, n (\%) & $13(15.1)$ \\
\hline $\begin{array}{l}\text { DOR median, months (range) } \\
(95 \% \mathrm{Cl})\end{array}$ & $\begin{array}{l}19.5(0.9-19.5) \\
(16.6-\mathrm{NE})\end{array}$ \\
\hline Follow-up median, months (range) & $18.4(0.3-23.5)$ \\
\hline $\begin{array}{l}\text { PFS median, months (range) } \\
(95 \% \mathrm{Cl})\end{array}$ & $\begin{array}{l}22.1(0.0-22.3) \\
(17.4-\mathrm{NE})\end{array}$ \\
\hline
\end{tabular}

In the BGB-3111-206 study, 86 patients with R/R MCL were enrolled. The IRC-assessed ORR was $84 \%$ with a median DOR of 19.5 months (Table 2) [21]. The most common adverse events were neutropenia (48.8\%), leukopenia and upper respiratory tract infection $(34.9 \%$ each), rash $(33.7 \%)$ and thrombocytopenia $(32.6 \%)$. The majority of AEs were grade 1/2; neutropenia was the most common grade $\geq 3$ AE (19.8\%). Eight patients (9\%) discontinued treatment due to AEs. Three patients $(3 \%)$ had major bleeding events. No patient experienced atrial fibrillation/flutter [21].

In a pooled safety analysis from six zanubrutinib monotherapy studies $(\mathrm{n}=682)$, including patients with NHL, the most common AEs that occurred in $\geq 20 \%$ of patients were upper respiratory tract infection and neutropenia [16]. The majority of AEs were grade 1/2, with neutrophil count decreased as the most common grade $\geq 3$ AE (14\%) [16]. Toxicities that are often associated with BTK inhibitors were infrequent, including grade $\geq 3$ atrial fibrillation/flutter $(0.6 \%)$, grade $\geq 3$ major hemorrhage $(2.1 \%)$, grade $\geq 3$ thrombocytopenia $(6.6 \%)$ and grade $\geq 3$ diarrhea $(0.9 \%)$. AEs leading to study drug discontinuation occurred in $9 \%$ of patients [22].

Rituximab, an anti-CD20 monoclonal antibody, is widely used in the treatment of different types of B-cell malignancies both as a single agent and in combination regimens. Rituximab has been demonstrated to be a key component in treatment of patients with MCL and is standardly used as part of frontline chemotherapy [5,23]. The addition of rituximab $\left(375 \mathrm{mg} / \mathrm{m}^{2}\right)$ to other agents in frontline treatment has shown an improvement in OS [24]. Clinical experiences with ibrutinib in combination with rituximab have demonstrated that the combinations are active, lack overlapping toxicity and can be combined at full monotherapy doses of the respective agents [25-27]. In preclinical studies, zanubrutinib was shown to be at least tenfold weaker than ibrutinib in inhibiting rituximabinduced antibody-dependent cell-mediated cytotoxicity, consistent with its more selective activity against BTK and weaker inducible T-cell kinase inhibitory activity than ibrutinib [28]. In preclinical xenograft models, zanubrutinib has also demonstrated good combination activity with rituximab. Since rituximab is a monoclonal antibody (biologic), no PK drug-drug interaction between zanubrutinib and rituximab is expected.

Given the encouraging clinical activity and tolerability of zanubrutinib in R/R MCL and the need for more frontline treatment options for the elderly and those with comorbidities, chemotherapy-free investigation of zanubrutinib in combination with rituximab for patients with previously untreated MCL who are ineligible for SCT is warranted.

\section{Design}

Study design

This Phase III, global, multicenter, randomized, open-label, active-control study compares zanubrutinib plus rituximab followed by zanubrutinib monotherapy versus bendamustine plus rituximab followed by observation in approximately 500 adult patients with previously untreated MCL who are ineligible for SCT. The primary objective of the study is to compare efficacy of the treatment arms, as measured by PFS determined by IRC.

This study opened to accrual in July 2019 and will be recruiting patients from approximately 200 sites in Australia, Austria, Belgium, China, France, Germany, Italy, Ireland, Japan, the Netherlands, New Zealand, Poland, Portugal, Romania, Russia, Spain, Taiwan, Turkey, Ukraine, UK and the USA. 


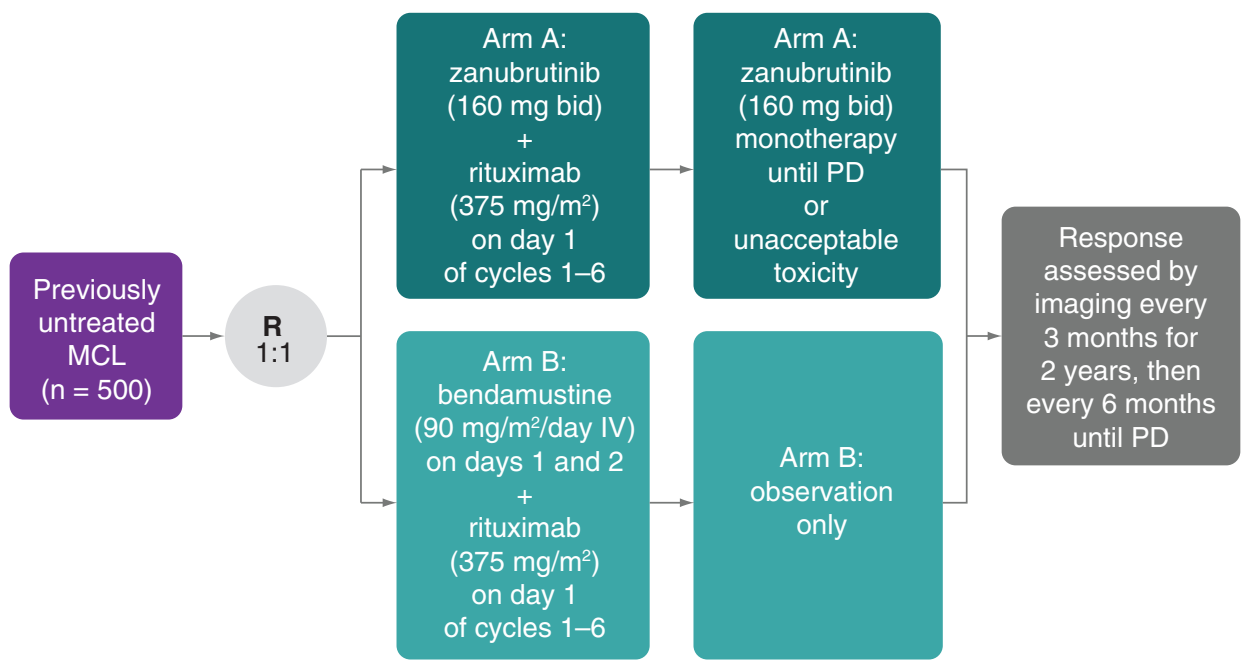

Figure 1. Study design.

Bid: Twice daily; MCL: Mantle cell lymphoma; PD: Progressive disease; R: Randomized.

\section{Key eligibility criteria}

Eligible patients must have histologically confirmed MCL with measurable disease by computed tomography/magnetic resonance imaging scan, an Eastern Cooperative Oncology Group performance status of 0-2 and adequate organ function. Patients must not have received prior systemic treatments for MCL. Patients must be ineligible for SCT, aged $\geq 70$ years, or aged $\geq 65$ and $<70$ years with comorbidities precluding autologous SCT including at least one of the following: cardiac ejection fraction $\leq 40 \%$, diffusing capacity for carbon monoxide $\leq 60 \%$ predicted and/or creatinine clearance $<70$ but $\geq 30 \mathrm{ml} / \mathrm{min}$.

Patients will be excluded for any of the following reasons: known central nervous system involvement by lymphoma, prior hematopoietic SCT, prior treatment with a BTK inhibitor, patients for whom the goal of therapy is tumor debulking prior to SCT, clinically significant cardiovascular disease, history of severe bleeding, known infection with HIV or active hepatitis $\mathrm{B}$ or $\mathrm{C}$ viruses, or prior malignancy within the past 3 years except for curatively treated basal or squamous cell skin cancer, superficial bladder cancer, carcinoma in situ of the cervix or breast, or localized Gleason score 6 prostate cancer.

\section{Planned sample size}

Approximately 500 patients will be enrolled and randomized in a 1:1 ratio to receive either zanubrutinib plus rituximab followed by zanubrutinib monotherapy or bendamustine plus rituximab followed by observation. Randomization will be stratified by age ( $<70$ vs $\geq 70$ years), geographic region (Asia Pacific vs North America/Europe) and MCL International Prognostic Index Score (low vs intermediate/high).

\section{Planned study period}

All study treatments will be administered open label. The first patient was dosed on 21 August 2019. Based on enrollment projections, the study duration is estimated to be approximately 84 months (7 years). Recruitment is ongoing.

\section{Study procedures}

Patients will receive either zanubrutinib $160 \mathrm{mg}(80 \mathrm{mg} \times$ two capsules) by mouth twice daily (with interval of $\geq 8$ hours) plus intravenous (iv.) rituximab $375 \mathrm{mg} / \mathrm{m}^{2}$ on day 1 of cycles $1-6$, followed by zanubrutinib monotherapy at the same dose (arm A) or iv. bendamustine $90 \mathrm{mg} / \mathrm{m}^{2} /$ day on days 1 and 2 plus iv. rituximab $375 \mathrm{mg} / \mathrm{m}^{2}$ on day 1 of cycles 1-6 followed by observation (arm B; Figure 1). Each treatment cycle consists of 28 days. Patients will remain on the study drugs until disease progression, unacceptable toxicity, treatment consent withdrawal, or study termination.

In the event of grade $\geq 3$ nonhematologic toxicities, grade $\geq 3$ febrile neutropenia, grade 3 thrombocytopenia associated with bleeding, or grade 4 thrombocytopenia or neutropenia lasting for more than ten consecutive days 
considered to be related to zanubrutinib, drug will be withheld until resolution of any related toxicities. Dosing can be resumed following an interruption of up to 28 days but at a reduced dose following the second and third $\mathrm{AE}(80 \mathrm{mg}$ twice daily and once daily, respectively). Zanubrutinib is to be discontinued in patients who experience their fourth drug-related AE. The local rituximab and bendamustine labels should be followed for the management of toxicities. In general, no dose reductions for rituximab will be allowed; a dose delay of up to 28 days is acceptable. In arm $\mathrm{B}$, if rituximab or bendamustine is delayed, then the initiation of the entire cycle should be delayed.

\section{Outcome measures/end points}

The primary objective of the study is to compare the efficacy of the treatment arms, as measured by PFS determined by IRC using the Lugano classification for NHL. Secondary objectives include PFS determined by IA, ORR (proportion of patients achieving complete response or partial response) determined by IRC and IA, DOR determined by IRC and IA, OS, rate of complete response or complete metabolic response, time to response determined by IRC and IA, patient-reported outcomes as measured by the 5-level EQ-5D (EQ-5D-5L) and the European Organization for the Research and Treatment of Cancer Quality of Life Questionnaire (EORTC QLQ C30) and safety parameters. Exploratory end points include PK parameters of zanubrutinib, a correlation analysis of clinical outcomes with prognostic and predictive biomarkers and an evaluation of the mechanisms of resistance to zanubrutinib.

Assessments of safety will include AEs, serious AEs, clinical laboratory tests, physical examinations, electrocardiograms and vital signs. AEs will be graded for severity per the National Cancer Institute Common Terminology Criteria for Adverse Events Version 5.0. An independent data monitoring committee will periodically monitor safety data and perform the interim efficacy analysis.

\section{Statistics}

The primary analysis set for all efficacy analyses is the intent-to-treat analysis set (all randomized patients). The safety analysis set will include all patients who receive any dose of study drug. The PK analysis set will include all zanubrutinib-treated patients who have at least one post-dose drug concentration measurement.

Approximately 500 patients will be enrolled and randomized in a 1:1 ratio to receive either zanubrutinib plus rituximab followed by zanubrutinib monotherapy or bendamustine plus rituximab followed by observation. The primary efficacy analysis comparing PFS assessed by IRC between the two arms will be based on a log-rank test stratified by randomization stratification factors age ( $<70$ vs $\geq 70$ years), geographic region (Asia Pacific vs North America/Europe) and MCL International Prognostic Index Score (low vs intermediate/high) in the intent-to-treat analysis set.

There will be planned analyses based on the number of PFS events as determined by IRC. The p-value will be based on Wald test for the treatment effect from the Cox regression. The hazard ratio and its two-sided $95 \%$ CI will be estimated from a stratified Cox regression model. The distribution of PFS, including median PFS and PFS rate at selected timepoints, will be estimated using the Kaplan-Meier method for each arm.

\section{Conclusion}

Targeting the BTK signaling pathway has proven to be efficacious in patients with R/R MCL. Zanubrutinib, a next-generation BTK inhibitor, has been shown to be highly active and well tolerated in R/R MCL. Rituximab has been a key component in the treatment of MCL. The combination of zanubrutinib with rituximab has the potential for improved outcomes in patients with previously untreated MCL who are ineligible for SCT and currently have limited treatment options. 
Executive summary

Background \& rationale

- Current frontline chemo-immunotherapeutic options can produce high response rates in mantle cell lymphoma (MCL) but relapse is inevitable for most patients.

- Furthermore, the elderly and those with comorbidities are precluded from standard regimens and stem cell transplantation (SCT), leaving them with limited treatment options.

- Targeting the B-cell receptor with Bruton tyrosine kinase (BTK) inhibitors, including zanubrutinib, has demonstrated clinical activity and has been approved by the US FDA in patients with relapsed/refractory MCL.

- Rituximab has demonstrated clinical benefits and has been stardardly used in first-line MCL patients.

Zanubrutinib

- Zanubrutinib is a next-generation irreversible BTK inhibitor designed to maximize BTK occupancy and minimize off-target inhibition of TEC- and EGFR-family kinases.

- In clinical studies of relapsed/refractory MCL, zanubrutinib monotherapy has been shown to be active and well tolerated.

Phase III trial

- This Phase III, global, multicenter, randomized, open-label, active-control study compares zanubrutinib plus rituximab followed by zanubrutinib monotherapy versus bendamustine plus rituximab followed by observation in approximately 500 adult patients with previously untreated MCL who are ineligible for SCT.

Objectives

- The primary objective of the study is to compare the efficacy of the treatment arms, as measured by progression-free survival (PFS) determined by an independent review committee.

- Key secondary end points include PFS determined by investigator assessment, overall response rate, duration of response, overall survival and safety.

Statistics

- The primary efficacy analysis comparing PFS assessed by independent review committee between the two arms will be based on a log-rank test stratified by randomization stratification factors age ( $<70$ vs $\geq 70$ years), geographic region (Asia Pacific vs North America/Europe) and MCL International Prognostic Index Score (low vs intermediate/high) in the intent-to-treat analysis set.

Conclusion

- Zanubrutinib is a next-generation BTK inhibitor designed to be highly selective with fewer off-target effects.

- Zanubrutinib was shown to be at least tenfold weaker than ibrutinib in inhibiting rituximab-induced antibody-dependent cell-mediated cytotoxicity consistent with its more selective activity against BTK.

- Zanubrutinib in combination with rituximab has the potential to further improve outcomes with a chemotherapy-free approach in patients with previously untreated MCL who are ineligible for SCT and currently have limited treatment options.

Author contributions

M Dreyling, CS Tam, M Wang, SD Smith, M Ladetto, H Huang and S Le Gouill have contributed or will contribute to patient enrollment. BeiGene was involved in study design, compilation of data and statistical analysis. All authors had full access to all of the data, contributed to data interpretation and analysis, carefully reviewed the manuscript and approved the final version. The corresponding author, M Dreyling, had final responsibility to submit for publication.

\section{Financial \& competing interests disclosure}

This clinical trial is supported by BeiGene USA, Inc., CA, USA. M Dreyling has received honoraria from Bayer, Celgene, Gilead, Janssen and Roche; served as consultant/advisor for Acerta, Bayer, Celgene, Gilead, Janssen, Novartis, Roche and Sandoz; and received research funding from Celgene, Janssen and Roche. CS Tam has served as consultant/advisor for BeiGene, Janssen, Roche, AbbVie and Loxo; and received research funding from Janssen, AbbVie, BeiGene, Pharmacyclics and TG Therapeutics. M Wang owns stock in MORE Health; has received honoraria from Pharmacyclics, Janssen, AstraZeneca, OMI, Targeted Oncology and OncLive; served as consultant/advisor for Pharmacyclics, Celgene, Janssen, AstraZeneca, MORE Health and Pulse; received research funding from Pharmacyclics, Janssen, AstraZeneca, Kite Pharma, Juno, Loxo Oncology, VelosBio and Celgene; and received travel/accommodation/expenses from Janssen, Pharmacyclics, Celgene, OMI, Kite Pharma and AstraZeneca. SD Smith has served as consultant/advisor for AstraZeneca and BeiGene; and received research funding from Acerta, AstraZeneca, Ayala (family member), Bristol Myers Squibb (family member), Genentech/Roche, Ignyta (family), Incyte, Merck Sharp \& Dohme, Pharmacyclics, Portola, Seattle Genetics, De Novo Pharmaceuticals and BeiGene. M Ladetto has received honoraria, research funding, travel/accommodations/expenses and has served as a consultant/advisor for AbbVie, Acerta, Amgen, Archigen, Celgene, ADC Therapeutics, Gilead, Novartis, Johnson \& Johnson, Roche, Roche Diagnostics, Sandoz, Takeda and BeiGene. H Huang has nothing 
to disclose. W Novotny is an employee of and owns stock in BeiGene. M Co is an employee of, owns stock in and received travel expenses from BeiGene. A Romano and E Holmgren are employees of and own stock in BeiGene. J Huang is an employee of, has a leadership role at and owns stock in BeiGene. S Le Gouill has received honoraria, travel/accommodations/expenses and has served as a consultant/advisor for AbbVie, Celgene, Gilead-Kite, Novartis, Janssen-Cilag, Roche, Roche Diagnostics, Servier, Daiichi Sankyo and Loxo. The authors have no other relevant affiliations or financial involvement with any organization or entity with a financial interest in or financial conflict with the subject matter or materials discussed in the manuscript apart from those disclosed.

Medical writing and editorial assistance was funded by BeiGene and was provided, under the direction of the authors, by KR Nibouar, Bio Connections, LLC.

\section{Ethical conduct of research}

The authors state that they have obtained appropriate institutional review board approval or have followed the principles outlined in the Declaration of Helsinki for all human or animal experimental investigations. In addition, for investigations involving human subjects, informed consent has been obtained from the participants involved.

\section{Open access}

This work is licensed under the Attribution-NonCommercial-NoDerivatives 4.0 Unported License. To view a copy of this license, visit http://creativecommons.org/licenses/by-nc-nd/4.0/

\section{References}

Papers of special note have been highlighted as: • of interest

1. Goy A. Update in the management of mantle cell lymphoma. Clin. Adv. Hematol. Oncol. 11(5), 297-299 (2013).

2. Skarbnik AP, Goy AH. Mantle cell lymphoma: state of the art. Clin. Adv. Hematol. Oncol. 13(1), 44-55 (2015).

3. Smedby KE, Hjalgrim H. Epidemiology and etiology of mantle cell lymphoma and other non-Hodgkin lymphoma subtypes. Semin. Cancer Biol. 21(5), 293-298 (2011).

4. Wang X-M, Bassig BA, Wen J-J et al. Clinical analysis of 1629 newly diagnosed malignant lymphomas in current residents of Sichuan province, China. Hematol. Oncol. 34(4), 193-199 (2016).

5. Dreyling M, Campo E, Hermine $\mathrm{O}$ et al. Newly diagnosed and relapsed mantle cell lymphoma: ESMO Clinical Practice guidelines for diagnosis, treatment and follow-up. Ann. Oncol. 28(S4), 62-71 (2017).

6. Sharman J, Black Shinn J, Clark J et al. MCL treatments and outcomes: a community oncology practice experience. Blood 130(Suppl. 1), 4684 (2017).

7. Jares P, Colomer D, Campo E. Molecular pathogenesis of mantle cell lymphoma. J. Clin. Invest. 122(10), 3416-3423 (2012).

8. Vose JM. Mantle cell lymphoma: 2017 update on diagnosis, risk-stratification and clinical management. Am. J. Hematol. 92(8), 806-813 (2017).

9. Kluin-Nelemans HC, Hoster E, Hermine O et al. Treatment of older patients with mantle-cell lymphoma. N. Engl. J. Med. 367(6), 520-531 (2012).

10. Rummel MJ, Niederle N, Maschmeyer G et al. Bendamustine plus rituximab versus CHOP plus rituximab as first-line treatment for patients with indolent and mantle-cell lymphomas: an open-label, multicentre, randomised, Phase 3 non-inferiority trial. Lancet 381(9873), 1203-1210 (2013).

11. Dreyling M, Lenz G, Hoster E et al. Early consolidation by myeloablative radiochemotherapy followed by autologous stem cell transplantation in first remission significantly prolongs progression-free survival in mantle-cell lymphoma: results of a prospective randomized trial of the European MCL Network. Blood 105(7), 2677-2684 (2005).

12. Romaguera JE, Fayad L, Rodriguez MA et al. High rate of durable remissions after treatment of newly diagnosed aggressive mantle-cell lymphoma with rituximab plus hyper-CVAD alternating with rituximab plus high-dose methotrexate and cytarabine. J. Clin. Oncol. 23(28), 7013-7023 (2005).

13. Aalipour A, Advani RH. Bruton tyrosine kinase inhibitors: a promising novel targeted treatment for B cell lymphomas. Br. J. Haematol. 163(4), 436-443 (2013).

14. Imbruvica (ibrutinib), package insert. Pharmacyclics, LLC, CA, USA. (2020).

15. Calquence (acalabrutinib), package insert. AstraZeneca Pharmaceuticals, LP, DE, USA. (2019).

16. Brukinsa (zanubrutinib), package insert. BeiGene USA, Inc, CA, USA. (2019).

17. Tam C, Grigg AP, Opat $S$ et al. The BTK inhibitor, BGB-3111, is safe, tolerable and highly active in patients with relapsed/refractory B-cell malignancies: initial report of a Phase 1 first-in-human trial. Blood 126(23), 832-832 (2015).

- Demonstrates complete blockade of Bruton tyrosine kinase (BTK) by zanubrutinib in peripheral blood mononuclear cells. 
18. Coutre SE, Byrd JC, Hillmen P et al. Long-term safety of single-agent ibrutinib in patients with chronic lymphocytic leukemia in 3 pivotal studies. Blood Adv. 3(12), 1799-1807 (2019).

19. Tam CSL, Trotman J, Opat $S$ et al. Phase 1 study of the selective BTK inhibitor zanubrutinib in B-cell malignancies and safety and efficacy evaluation in CLL. Blood 134(11), 851-859 (2019).

20. Tam C, Wang M, Simpson D et al. Updated safety and efficacy data in the Phase 1 trial of patients with mantle cell lymphoma (MCL) treated with Bruton tyrosine kinase (BTK) inhibitor zanubrutinib (BGB-3111). Hematol. Oncol. 37(S2), Abstract 191 (2019).

- Single-arm study demonstrating activity and tolerability of zanubrutinib monotherapy in patients with mantle cell lymphoma.

21. Song Y, Zhou K-S, Zou D et al. Treatment of patients with relapsed or refractory mantle cell lymphoma with zanubrutinib, a selective inhibitor of Bruton's tyrosine kinase. Clin. Cancer Res. 26(16), 4216-4224 (2020).

- This single-arm study demonstrats the activity and tolerability of zanubrutinib monotherapy in patients with mantle cell lymphoma.

22. Tam C, Opat S, Zhu J et al. Pooled analysis of safety data from monotherapy studies of the Bruton tyrosine kinase (BTK) inhibitor, zanubrutinib (BGB-3111) in B-cell malignancies. HemaSphere 3(S1), Abstract PS1159 (2019).

- Demonstrates the low rate of BTK inhibitor-associated toxicities, including atrial fibrillation/flutter.

23. National Comprehensive Cancer Network. NCCN clinical practice guidelines in oncology - B-cell lymphomas. Version 5. (2018).

24. Schulz H, Bohlius JF, Trelle $S$ et al. Immunochemotherapy with rituximab and overall survival in patients with indolent or mantle cell lymphoma: a systematic review and meta-analysis. J. Natl Cancer Inst. 99(9), 706-714 (2007).

25. Wang ML, Lee $\mathrm{H}$, Chuang $\mathrm{H}$ et al. Ibrutinib in combination with rituximab in relapsed or refractory mantle cell lymphoma: a single-centre, open-label, Phase II trial. Lancet Oncol. 17(1), 48-56 (2016).

- Demonstrates the feasibility of combining a BTK inhibitor with rituximab.

26. Burger J, Hartmann E, Hoellenriegel J et al. Safety and activity of ibrutinib plus rituximab for patients with high-risk chronic lymphocytic leukaemia: a single-arm, Phase 2 study. Lancet Oncol. 15(10), 1090-1099 (2014).

27. Fowler N, Nastoupil L, De Vos S et al. Ibrutinib combined with rituximab in treatment-naive patients with follicular lymphoma: arm $1+\operatorname{arm} 2$ results from a multicenter, open-label Phase 2 study. Blood 128(22), 1804-1804 (2016).

28. Guo Y, Liu Y, Hu N et al. Discovery of zanubrutinib (BGB-3111), a novel, potent and selective covalent inhibitor of Bruton's tyrosine kinase. J. Med. Chem. 62(17), 7923-7940 (2019). 